\title{
Revisión
}

\section{Fibrilación atrial: Nuevos conceptos}

\author{
Luis Gutiérrez-Jaikel ', Mario Bolaños-Arrieta 2, Oswaldo Gutiérrez-Sotelo ${ }^{3}$.
}

\section{R e s u m e n}

La fibrilación atrial es la arritmia más frecuente en la práctica clínica ${ }^{1-12}$, se presenta tanto en pacientes con cardiopatía estructural de base principalmente hipertensiva, isquémica y valvular, como en pacientes sin trastorno cardiaco de fondo al avanzar la edad. Debido a la morbilidad asociada a esta, es vital su reconocimiento temprano y el manejo adecuado, con la finalidad de reducir la incidencia de complicaciones asociadas y mejorar la calidad de vida de quienes la padecen.

Descriptores: arritmia, cardioversión, anticoagulación, cardiopatía, automatismo, reentrada.

Recibido: 26 de septiembre de 2003 Aceptado:09 de marzo de 2004

\section{HISTORIA}

Desde época inmemorial se han dado diversos nombres a la FA: palpitaciones rebeldes, delirium cordis, pulsus irregularis perpetuus, entre otros. Einthoven, en 1906, hizo la primera demostración electrocardiográfica de este fenómeno. Más tarde, informes simultáneos de Lewis, Rothberger y Winterberg, confirmaron la relación entre la fibrilación auricular corroborada por electrocardiografía y el cuadro clínico de pulso "irregularmente irregular" crónico1

\section{DEFINICIÓN}

1. Médico general.

2. Cardiólogo asistente del Hospital San Rafael de Alajuela.

3. Cardiólogo y electrofisiólogo asistente del Hospital México.

Abreviaturas: FA; Fibrilación atrial, ICC; insuficiencia cardiaca congestiva, INR; índice internacional normalizado, HDL; high density lipoproteins

Correspondencia: Luis Gutiérrez Jaikel, apartado postal 88-4050, Alajuela, Costa Rica, Fax: 443-|8-4I.

Correo electrónico: gjaikel@hotmail.com

ISSN 0001-6002/2004/46/2/58-67 Acta Médica Costarricense, (C2004 Colegio de Médicos y Cirujanos
La FA consiste en una taquiarritmia por disparo rápido e irregular de ondas fibrilatorias que varían en tamaño y amplitud desde focos atriales simultáneos, o por reentrada mediante vías internodales a una frecuencia de 350 a 600 por minuto ${ }^{1,13,14}$, asociado a una respuesta ventricular irregular. Desde el punto de vista electrocardiográfico existe ausencia de ondas $\mathrm{P}$, presencia de ondas $\mathrm{f}$ más visibles en la derivación V1 y un intervalo R-R irregular; el QRS es de amplitud normal ${ }^{1,15}$, excepto cuando existe conducción aberrante o un bloqueo de rama preexistente.

\section{EPIDEMIOLOGÍA}

La incidencia de la FA en la población aumenta de forma indiscutible al aumentar la edad $1,2,13,16,17$ se observan 2 ó 3 casos nuevos por cada 1000 entre los 55 y 64 años y 35 casos nuevos por cada 1000 entre los 85 y 94 años de edad ${ }^{5}$. Además de ser la arritmia más común en la práctica clínica ${ }^{1-12,17-22}$ es un importante factor de riesgo para enfermedad cerebrovascular isquémical ${ }^{5-7,12,15,16,23}$, así como para enfermedad vascular periférica de origen embólico, con un factor predisponente para descompensación de la insuficiencia cardiaca. Se presenta en forma aislada en el $3 \%$ de los casos y se considera de buen pronóstico en pacientes menores de 60 años de edad. ${ }^{13}$

La FA es la forma de presentación clínica de entre el 5\% y el $15 \%$ de los pacientes con hipertiroidismo, ${ }^{24}$ principalmente mujeres. 


\section{ETIOLOGÍA}

La FA puede deberse a un gran número de causas que se clasifican en 2 grupos, cardiacas y sistémicas. (Cuadro 1)

\begin{tabular}{|c|c|}
\hline \multicolumn{2}{|c|}{ Cuadro 1: Causas de fibrilación auricular } \\
\hline $\begin{array}{l}\text { Cardiacas: } \\
\text { - Cirugía cardiaca } \\
\text { - Miocardiopatías } \\
\text { - Cardiopatía congénita } \\
\text { - Hipertensión } \\
\text { - Cardiopatía isquémica } \\
\text { - Pericarditis } \\
\text { - } \text { Hipertrofia lipomatosa } \\
\text { - Tumores de prexcitación } \\
\text { - Valvulopatías } \\
\text { - Hipertrofia ventricular } \\
\text { - Marcapaso } \\
\text { - Síndrome taquicardia - bradicardia }\end{array}$ & $\begin{array}{l}\text { Sistémicas: } \\
\text { - Edad } \\
\text { - Alcohol } \\
\text { - EPOC } \\
\text { - Electrolitos } \\
\text { - Fiebre } \\
\text { - Hipotermia } \\
\text { - Hipovolemia } \\
\text { - Embarazo } \\
\text { - Deglución } \\
\text { - Tirotoxicosis } \\
\text { - Traumatismo } \\
\text { - Enfermedad cerebrovascular } \\
\text { - Cambios emocionales repentinos }\end{array}$ \\
\hline
\end{tabular}

te del periodo refractario, ${ }^{7,8,12,25}$ lo cual favorece la reentrada. Los impulsos auriculares son bloqueados por colisión de ondas o por la unión atrio-ventricular. ${ }^{13}$

Electrocardiográficamente encontramos:

- Oscilación irregular de la línea de base del electrocardiograma.

- Ausencia de ondas P.

- Intervalo R-R irregular.

- Ondas f, más evidentes en V114 (pueden no observarse).

- La respuesta ventricular es variable, siendo baja cuando es menor de 60 latidos por minuto, adecuada entre 60 y 100 , o alta cuando es mayor de 100 .

- QRS de amplitud normal, excepto cuando existe conducción aberrante por una vía accesoria o bloqueo de rama preexistente.

\section{CLASIFICACIÓN}

La FA puede ser de tres tipos, según la forma de presentación y su duración. La FA paroxística es aquella que se presenta como episodios autolimitados de menos de 48 horas de evolución, con retorno al ritmo sinusal de forma espontánea; la FA persistente es aquella que se prolonga por más de 48 horas y en la cual el ritmo sinusal no se reestablece de forma espontánea, pero la cardioversión farmacológica o eléctrica está indicada y reestablece el ritmo sinusal. La FA permanente o crónica es aquella en la cual no es posible reestablecer el ritmo sinusal, a pesar de la adecuada cardioversión farmacológica y eléctrica, indistintamente del tiempo de evolución. ${ }^{18,25,26}$

\section{ELECTROFISIOLOGÍA}

En la mayoría de los casos la FA es secundaria a la existencia de múltiples circuitos pequeños de reentrada en el atrio $1,13,14$. Otro mecanismo posible es la presencia de focos ectópicos múltiples que generan impulsos a una velocidad excesiva (automatismo aumentado) ${ }^{19,26}$. Recientemente se han encontrado focos ectópicos en extensiones de tejido atrial, en las venas pulmonares y uniones cavoatriales ${ }^{27}$.

Se pueden considerar como predisponentes al desencadenamiento de la FA, las alteraciones en el tono del sistema nervioso autónomo, los cambios en la presión parietal atrial, la cirugía de tórax, los focos de ectopia en el atrio, las alteraciones electrolíticas o metabólicas y algunos fármacos.

Desde el punto de vista eléctrico, la arritmia tiende a autoperpetuarse por medio de un mecanismo llamado remodelado electrofisiológico, que consiste en un acortamiento permanen-

\section{EFECTOS HEMODINÁMICOS}

La ausencia de sístole atrial reduce el volumen de llenado ventricular, y por ende, el gasto cardiaco hasta en un $20 \%^{13}$, esto unido a la presencia de una respuesta ventricular rápida, reduce aún más el llenado ventricular. Los factores mencionados pueden ser críticos en pacientes con estenosis de la válvula mitral, en los cuales la sístole atrial es esencial en el llenado ventricular, razón por la cual la FA puede desencadenar en ellos un edema agudo de pulmón.

Cuando se tiene una respuesta ventricular rápida, la caída secundaria del gasto cardiaco puede inducir hipotensión arterial y síncope, así como congestión pulmonar y angor en pacientes predispuestos. Estos efectos parecen ser muy marcados en pacientes con miocardio hipertrófico y disfunción diastólica, como es el caso de individuos con miocardiopatía hipertrófica, cardiopatía hipertensiva y miocardiopatías restrictivas (sarcoidosis, hemocromatosis, amiloidosis). Además, la FA puede inducir una taquimiocardiopatía secundaria a una frecuencia cardiaca persistentemente elevada ${ }^{2,16,26}$.

\section{CLÍNICA}

\section{Síntomas}

La FA puede ser mínimamente sintomática o asintomática en muchos pacientes ${ }^{1,13,16}$; en caso contrario, la sintomatología es producida por la arritmia y los cambios hemodinámicos que esta produce, o secundaria a embolismo sistémico.

La sintomatología relacionada con la arritmia y las alteraciones hemodinámicas que ésta causa incluye las palpitaciones y ansiedad secundaria, el mareo, la lipotimia, el síncope, la fatiga persistente y fatigabilidad fácil, las alteraciones en la función cognoscitiva principalmente en adultos mayores ${ }^{13}$, el 
empeoramiento de la ICC, la muerte súbita en pacientes con síndrome de Wolf-Parkinson-White ${ }^{28}$, la angina en pacientes con enfermedad arterial coronaria o miocardiopatía hipertrófica, disnea de esfuerzo o de reposo secundaria a congestión pulmonar e incluso edema pulmonar agudo.

Los síntomas relacionados con embolismo sistémico incluyen las embolias a extremidades y vísceras, la isquemia cerebral transitoria, el déficit isquémico cerebral reversible y el infarto cerebral isquémico, cuya sintomatología dependerá del territorio vascular comprometido.

\section{Signos clínicos}

Entre los hallazgos más comunes durante la exploración física del paciente con FA se encuentran: ausencia de onda “a" del pulso venoso ${ }^{29-31}$, pulso arterial irregular generalmente con taquicardia, primer ruido cardiaco de intensidad variable, datos de insuficiencia cardiaca o edema agudo de pulmón, chasquido de apertura con retumbo mesodiastólico en los pacientes con estenosis mitral (el reforzamiento presistólico no se auscultará por la ausencia de sístole atrial) y déficit del pulso en pacientes con contracciones ventriculares, sin intensidad suficiente para producir apertura de la válvula aórtica ${ }^{29}$.

\section{ABORDAJE DEL PACIENTE ${ }^{32}$}

\section{Historia clínica y exploración física}

En los pacientes con FA debe detallarse la presencia de síntomas y su naturaleza, interrogar sobre la existencia de episodios previos de FA sintomática o asintomática definiendo la fecha del primer episodio, la duración de los episodios previos, los precipitantes y la forma de terminación de la arritmia en cada episodio, ya sea espontáneamente o mediante cardioversión eléctrica o farmacológica.

Además, debe definirse la presencia de cardiopatía subyacente, ya sea hipertensiva, valvular, isquémica, miocardiopatía de cualquier etiología e ICC, así como de factores relacionados (alcoholismo, diabetes, hipertiroidismo, alteraciones hidroelectrolíticas y ansiedad, entre otros).

\section{ECG}

En el electrocardiograma debe valorarse la respuesta ventricular, la presencia de criterios electrocardiográficos de hipertrofia ventricular derecha o izquierda, datos de infarto previo o isquemia actual, valorar la presencia de bloqueos de rama preexistentes, y en caso de que se reestablezca el ritmo sinusal, analizar la duración y la morfología de la onda P.

\section{Exámenes de laboratorio y gabinete:}

En el estudio de todo paciente con FA se sugiere realizar siempre los siguientes exámenes de laboratorio y gabinete:

1. Hemograma completo.
2. Bioquímica: glucosa, colesterol total, HDL-colesterol, LDL-colesterol triglicéridos, urea, creatinina, electrolitos (sodio, potasio, calcio, magnesio).

3. Función Tiroidea: la cual debe ser considerada en todo paciente con FA de reciente descubrimiento, principalmente si es mujer, si la respuesta ventricular es difícil de controlar, si el paciente ha recibido tratamiento previo con amiodarona o en los pacientes que presenten signos y síntomas de hipertiroidismo.

4. Radiografía de tórax.

5. Electrocardiograma.

\section{Ecocardiograma:}

a. Transtorácico: todo paciente que se presente con un primer episodio de FA debe tener un ecocardiograma transtorácico de base, el cual permite determinar si es portador de una cardiopatía estructural de base, entre las cuales cabe mencionar, por su elevada frecuencia, la cardiopatía hipertensiva, la valvulopatía mitral, la cardiopatía isquémica, el cor pulmonale y los trastornos del pericardio. Los datos que de este se deriven, permiten guiar la escogencia de los fármacos de control, porque muchos están contraindicados en caso de disfunción sistólica ventricular ${ }^{6}$.

Dentro de las ventajas de la ecocardiografía transtorácica está la determinación del tamaño del atrio izquierdo, determinación de la función ventricular global y la posibilidad de obtener el diagnóstico de cardiopatía asociada. La principal desventaja es la imposibilidad de observar los apéndices auriculares, sitio de origen de la mayoría de los trombos $(90 \%)^{6}$.

b. Transesofágico: Esta técnica de imagen brinda una excelente visualización de los apéndices auriculares, lo cual permite descartar con altos índices de sensibilidad la presencia de trombos. Si se demuestra la ausencia de trombos en los apéndices auriculares, se puede realizar cardioversión temprana, favoreciendo la recuperación mecánica del atrio y disminuyendo las recurrencias al reducir el tiempo de exposición a la arritmia, factor que se relaciona con la severidad del remodelado mecánico y eléctrico del atrio. Sus principales desventajas son su invasividad, necesidad de un operador experimentado y costos más elevados.

\section{TRATAMIENTO}

\section{Cardioversión farmacológica a ritmo sinusal}

Antes de iniciar cualquier tratamiento para una FA de inicio reciente se debe valorar el riesgo/beneficio, debido a que entre el $44 \%$ y el $78 \%$ de los pacientes con FA de menos de 24 horas sufren cardioversión espontánea ${ }^{33}$.

La cardioversión farmacológica produce alivio sintomático y hemodinámica, además de reducir el riesgo embólico. El tratamiento medicamentoso siempre está indicado en la 
FA que no se autolimita en un paciente hemodinámicamente estable; los fármacos de elección son los antiarrítmicos de clase IC ${ }^{32,34}$.

\section{Fármacos utilizados (ver cuadros 2 y 3):}

\section{Antiarrítmicos de clase IA:}

La quinidina es un fármaco de uso extendido en la cardioversión farmacológica, pero su uso debe ser cuidadoso, debido a que por sus propiedades vagolíticas puede elevar paradójicamente la frecuencia cardiaca; la eficacia de este fármaco para restablecer el ritmo sinusal oscila entre el $40 \%$ y el $86 \%{ }^{34}$ Estudios comparativos han demostrado que la procainamida es igualmente efectiva. ${ }^{33}$

Con la quinidina y en general con los fármacos de clase IA, debe tenerse presente el riesgo de inducción de taquicardia helicoidal, debido a la prolongación del intervalo Q-T y prolongación del periodo refractario, en cuyo caso el tratamiento por elegir es el sulfato de magnesio.

\section{Antiarrítmicos de clase IC:}

Los medicamentos de este grupo incluyen la propafenona y la flecainida, cuyos mecanismos de acción consisten en el bloqueo de los canales de sodio prolongando el QRS sin afectar el Q-T. Las tasas de éxito llegan hasta el 90\%, pero se reducen al prolongarse el tiempo de evolución de la FA 34. Este grupo farmacológico es de elección si el paciente no posee cardiopatía estructural de base.

Durante el tratamiento debe siempre vigilarse el QRS el cual no debe ensancharse más de un $50 \%$ del basal ${ }^{32}$, este grupo no posee como efecto adverso la inducción de taquicardia helicoidal debido a que no prolonga el Q-T.

\section{Antiarrítmicos de clase III:}

Este grupo incluye el sotalol, el bretilio y la amiodarona. Los índices de éxito para la cardioversión son bajos excepto para la amiodarona, pero de gran utilidad para la prevención de recurrencias de la FA. El sotalol logra cardiovertir entre el $8 \%$ y el $54 \%$, y la amiodarona entre el $47 \%$ y el $93 \%$. Otras drogas más recientes como son el ibutilide y el dofetilide demostraron, índices de conversión a ritmo sinusal del $60 \%{ }^{36,37}$.

\section{Fármacos contraindicados y elección de antiarrítmicos en pacientes con FA, según patología de base del paciente}

- Insuficiencia cardiaca: están contraindicados los fármacos de clase I y III excepto la amiodarona, considerada de elección. Los betabloqueadores y los calcio-antagonistas deben ser usados con precaución en estos pacientes.

- Enfermedad arterial coronaria: se contraindican los fármacos de clase I y el fármaco de elección es el sotalol. Los betabloqueadores y los antagonistas del calcio son también opciones adecuadas.
- Hipertrofia ventricular izquierda: no se recomiendan los fármacos de clase III, excepto la amiodarona y el sotalol. Cuando el QRS es de duración normal, el medicamento de elección es la flecainida, pero cuando el QRS está prolongado o hay alteraciones de la onda $\mathrm{T}$ o segmento $\mathrm{S}-\mathrm{T}$, el sotalol es el fármaco indicado. Los betabloqueadores y los antagonistas del calcio son también opciones adecuadas.

- Intervalo Q-T prolongado: no deben utilizarse los fármacos de clase I y III; pueden usarse los antiarrítmicos de clase II y IV.

- Síndrome de Wolf-Parkinson-White: está contraindicado el uso del verapamilo y la digoxina; no existe contraindicación para el uso de otras clases de antiarrítmicos.

La digoxina, a pesar de que es ampliamente utilizada para el control de la respuesta ventricular, no se considera de elección y es mucho menos efectiva que las otras opciones terapéuticas disponibles para el control de la respuesta ventricular, siendo más efectivos los betabloqueadores y los antagonistas del calcio. Actualmente su principal indicación es en pacientes con insuficiencia cardiaca por disfunción sistólica.

\section{Prevención de recurrencias:}

Existen 3 opciones de acción, según los mecanismos electrofisiológicos, entre los cuales se encuentra el uso de fármacos que evitan la elevada respuesta ventricular, el empleo de medicamentos que eliminan la ectopia atrial (automatismo) y la utilización de fármacos que prolongan el periodo refractario.

Actualmente han surgido los conceptos de FA dependiente de catecolaminas y vago-dependiente. Los pacientes con cardiopatía estructural, por lo general poseen una arritmia dependiente de catecolaminas, siendo en ellos ideal el control de su enfermedad base y el uso de bloqueadores de los receptores beta. Los pacientes jóvenes sin cardiopatía, con frecuencia poseen una FA vagal. En este último grupo de pacientes no deben utilizarse bloqueadores beta ni digoxina, siendo en ellos de elección la disopiramida, la flecainida y la quinidina por su efecto vagolítico, medicamentos también considerados de utilidad en la prevención de recurrencias.

Las recurrencia de la FA tiende a presentarse con mayor frecuencia en pacientes con arritmia de duración prolongada (1-3 años), atrio izquierdo de más de $55 \mathrm{~mm}$, edad mayor a 60 años y cardiopatía reumática ${ }^{6,832}$.

Los fármacos más eficaces para mantener a los pacientes en ritmo sinusal son los antiarrítmicos de clase III, particularmente la amiodarona y el sotalol. En los pacientes sin cardiopatía estructural son útiles los de clase IC (flecainida y propafenona) en conjunto con una droga para el control de la respuesta ventricular ${ }^{34}$. 


\begin{tabular}{|c|c|c|}
\hline Fármaco & Dosis para cardioversión & Dosis de mantenimiento \\
\hline Flecainida & 300 mg vía oral & 50-150 mg dos veces al día \\
\hline Propafenona & $600 \mathrm{mg}$ vía oral & $150-300 \mathrm{mg}$ dos veces al día \\
\hline Procainamida & $\begin{array}{l}100 \text { mg intravenoso cada } \\
5 \text { minutos, máximo }\end{array}$ & $\begin{array}{l}1000 \mathrm{mg} \mathrm{1000-2000} \mathrm{mg} \\
\text { dos veces al día }\end{array}$ \\
\hline Quinidina & $\begin{array}{l}200 \text { mg vía oral, luego } 400 \\
\text { mg } 1 \text { ó } 2 \text { horas después }\end{array}$ & $200-400$ mg cuatro veces al día \\
\hline Disopiramida & $\begin{array}{l}200 \text { mg vía oral cada } 4 \text { horas } \\
\text { hasta } 800 \mathrm{mg}\end{array}$ & 100-150 mg cuatro veces al día \\
\hline Sotalol & No recomendado & 120-160 mg dos veces al día \\
\hline Dofetilide & $\begin{array}{l}0.5 \text { mg vía oral (ajustar en } \\
\text { insuficiencia renal) }\end{array}$ & $\begin{array}{l}0.5 \mathrm{mg} \text { dos veces al día } \\
\text { (ajustar en insuficiencia renal) }\end{array}$ \\
\hline Amiodarona & $\begin{array}{l}1200 \text { mg intravenoso en } 24 \\
\text { horas }\end{array}$ & $\begin{array}{l}600 \text { mg por día/2 semanas, } \\
\text { luego 200-400 mg por día }\end{array}$ \\
\hline Ibutilide & $\begin{array}{l}1 \text { mg intravenoso en } 10 \\
\text { minutos, puede repetirse una } \\
\text { vez más si no hay respuesta }\end{array}$ & No disponible \\
\hline
\end{tabular}

Modificado de referencia 13

\begin{tabular}{|c|c|c|}
\hline Fármaco & Dosis para manejo agudo & Dosis para mantenimiento \\
\hline Propanolol & $\begin{array}{l}1-5 \mathrm{mg} \text { intravenoso en } \\
10 \text { minutos. }\end{array}$ & $30-360 \mathrm{mg}$ en dosis divididas \\
\hline Esmolol & $\begin{array}{l}0.5 \mathrm{mg} / \mathrm{kg} \text { intravenoso, repetir } \\
\text { en caso necesario, luego } \\
\text { infusión a } 0.05 \mathrm{mg} / \mathrm{kg} / \mathrm{min} \text {, } \\
\text { aumentar hasta } 0.2 \mathrm{mg} / \mathrm{kg} / \mathrm{min} \text {. }\end{array}$ & No disponible \\
\hline Metoprolol & $\begin{array}{l}5 \text { mg intravenoso en bolo, } \\
\text { repetir dos veces en intervalos } \\
\text { de } 2 \text { minutos. }\end{array}$ & 50-400 mg por día dividido \\
\hline Diltiazem & $\begin{array}{l}20 \mathrm{mg} \text { en bolo intravenoso, } \\
\text { luego infusión de } 5-15 \mathrm{mg} / \mathrm{hora} \text {. }\end{array}$ & $\begin{array}{l}\text { 180-300 mg por día en formulación } \\
\text { de liberación prolongada }\end{array}$ \\
\hline Verapamil & $\begin{array}{l}5-10 \text { mg intravenoso en } \\
2-3 \text { minutos, repetir una vez en } \\
30 \text { minutos. }\end{array}$ & $\begin{array}{l}120-240 \text { mg por día o dos veces } \\
\text { al día en formulación de } \\
\text { liberación prolongada }\end{array}$ \\
\hline Digoxina & $\begin{array}{l}1.0-1.5 \mathrm{mg} \text { intravenoso o oral } \\
\text { en } 24 \text { horas en dosis de } \\
0.25-0.5 \mathrm{mg} \text {. }\end{array}$ & 0.125-0.5 mg por día \\
\hline
\end{tabular}

Modificado de referencia 13
Además de la prevención de las recurrencias, es importante el control de la respuesta ventricular, tanto durante el reposo como en el ejercicio, los fármacos que con mayor frecuencia son utilizados para este fin son la digoxina, los antagonistas de los canales de calcio no dihidropiridinas (verapamila y diltiazem), los bloqueadores beta y la amiodarona (ver cuadro 3). El digital se considera de elección en pacientes con insuficiencia cardiaca por disfunción sistólica ${ }^{16}$, y en el caso de la amiodarona siempre deben tenerse presentes sus efectos adversos potenciales, tales como: agravamiento de la insuficiencia cardiaca, bradicardia sintomática y bloqueo atrioventricular, depósito corneal del fármaco, fibrosis pulmonar, pigmentación cutánea azulada, fotodermatitis, parestesias, tremor, ataxia, cefalea, la disfunción tiroidea, estreñimiento y hepatitis. ${ }^{34}$

\section{Control del ritmo versus control de la respuesta ventricular en FA}

Después de la cardioversión, sólo el $30 \%$ de los pacientes se mantienen en ritmo sinusal durante el primer año ${ }^{2}$. Esto implica elevados costos en salud por reingreso hospitalario. El estudio de intervención farmacológica en fibrilación atrial (PIAF), en el cual a un grupo de pacientes se le asignó anticoagulación y diltiazem para el control de la frecuencia cardiaca y al otro, cardioversión eléctrica y amiodarona para el control del ritmo, demostró una mejoría sintomática semejante en ambos. ${ }^{2}$

El estudio incluyó pacientes de entre 18 y 75 años, con FA sintomática de 7 y 360 días de evolución. Fueron excluidos pacientes con ICC, clase funcional IV, angor inestable, infarto en los 30 días previos, FA con frecuencia cardiaca menor de 50 por minuto, enfermedad del nodo sinusal, pacientes con síndrome de Wolf-Parkinson-White, bypass coronario o reemplazo valvular en los tres meses previos, miocardiopatía hipertrófica, tratamiento con amiodarona en los 6 meses previos, disfunción tiroidea aguda, marcapaso, contraindicaciones para anticoagulación o embarazo. ${ }^{2}$

Los resultados de este estudio demuestran que ninguna de las 2 opciones planteadas es superior para disminuir los síntomas relacionados con la $\mathrm{FA}^{2}$. Debe, sin embargo, tenerse presente que el estudio solo a pacientes con corazón estructuralmente sano, lo cual excluye a una gran parte de los pacientes con FA. Otros hallazgos del estudio consisten en la mejoría de la tolerancia al ejercicio en los pacientes asignados a control de ritmo, 
pero este mismo grupo asoció más hospitalizaciones y más efectos adversos al tratamiento, relacionados posiblemente con el uso de la amiodarona.

Otro estudio llamado Atrial Fibrillation Follow-up Investigation of Rythm Management (AFFIRM), comparó control del ritmo y control de respuesta ventricular en pacientes con FA paroxística o persistente. En el estudio, los pacientes aleatorizados a control del ritmo requirieron hospitalización más frecuentemente y se demostró que ambas estrategias son iguales en el punto final del estudio: la mortalidad a 1 año de seguimiento. El riesgo de embolia fue bajo con un $1 \%$ por año, en ambos grupos ${ }^{12}$, y la mayoría de los eventos se produjeron en pacientes que no estaban tomando warfarina o con INR subóptimo ${ }^{20}$.

\section{Anticoagulación}

Debido al elevado riesgo cardioembólico asociado con la arritmia, la anticoagulación es parte integral del tratamiento y para ello el fármaco de elección es la warfarina, que demostró reducir dramáticamente el riesgo de tromboembolismo ${ }^{38}$.

Como consecuencia de la posibilidad de sangrado asociado a la anticoagulación, se propuso el uso de aspirina aislada o asociada a warfarina a dosis bajas, con la finalidad de reducir la incidencia de enfermedad cerebrovascular isquémica sin elevar el riesgo de sangrado, sin embargo, los reanálisis de datos no demostraron beneficio significativo de esta estrategia para la prevención del ictus ${ }^{5,39}$.

El estudio Stroke Prevention in Atrial Fibrillation III randomized trial (SPAF III), demostró que los eventos cerebrovasculares primarios fueron mucho más frecuentes en pacientes con terapia de combinación con aspirina y warfarina a dosis bajas, en comparación con warfarina a dosis ajustada para un INR de $2-3$ (7.9\% vs $1.9 \%$ por año) ${ }^{39}$. Los resultados de este estudio y el beneficio con warfarina con un INR entre 2-3 ${ }^{40,42}$ involucran pacientes con factores de riesgo identificables para tromboembolismo (ver cuadro 4).

La warfarina es superior a otras terapias y este beneficio se debe a que los pacientes en los que no existen condiciones que incrementen la posibilidad de tromboembolismo, tienen un índice de embolias del $1 \%$ por año; mientras que los pacientes con 3 o más factores presentan un índice de embolias del $19 \%$ anual ${ }^{41,42}$. Por lo tanto, en los pacientes menores de 65 años que no presentan otras condiciones asociadas, el tratamiento antitrombótico con aspirina es adecuado para reducir la posibilidad de ictus isquémico (ver cuadro 5), opción que puede ser también considerada en pacientes en los que la warfarina esté contraindicada, por ejemplo:

Sangrado gastrointestinal o genitourinario en los 6 meses previos; caídas frecuentes o severas; incapacidad para seguir el tratamiento; uso diario de antinflamatorios no esteroideos; hipertensión arterial no controlada (mayor a 180/100; embarazo; ingesta de alcohol mayor a 14 bebidas por semana.
Estas contraindicaciones deben tenerse presentes, debido a que la mayoría de los eventos hemorrágicos relacionados con la terapia, se producen en pacientes mal seleccionados, uno o más factores de riesgo. Por otra parte, debe recalcarse que a pesar de la gran morbimortalidad asociada a los eventos embólicos, solo el 23\% de los pacientes con FA están anticoagulados, mientras que entre el $30 \%$ y el $50 \%$ se beneficiarían de la anticoagulación ${ }^{42}$. En el caso de los pacientes con FA de origen valvular, por el elevado riesgo tromboembólico, existe consenso general sobre la indicación de anticoagulación con warfarina en ausencia de contraindicación absoluta ${ }^{5,19}$, además, actualmente se sabe que tanto la FA paroxística como la persistente, presentan igual riesgo embolígeno ${ }^{5}$, por ende la warfarina será de elección en ambos casos si el paciente presenta alguno de los factores de riesgo mencionados.

\begin{tabular}{|l|}
\multicolumn{1}{c|}{$\begin{array}{c}\text { Cuadro 4. Factores de riesgo para } \\
\text { tromboembolismo sistémico }{ }^{12}\end{array}$} \\
\hline Edad \\
Hipertensión arterial \\
Embolismo previo \\
Diabetes mellitus \\
Insuficiencia cardiaca o fracción de eyección menor al 25\% \\
Hipertiroidismo \\
Sexo femenino \\
Hipercoagulabilidad \\
Tamaño del atrio mayor a 55 mm \\
Disfunción ventricular izquierda \\
Calcificación mitral \\
Trombo en el atrio izquierdo \\
Humo en el atrio izquierdo en el ecocardiograma transesofágico \\
Pobre función de transporte del apéndice del atrio izquierdo, \\
luego de la cardioversión
\end{tabular}

\begin{tabular}{|lll|}
\hline $\begin{array}{c}\text { Cuadro 5. Recomendaciones para anticoagulación } \\
\text { y tratamiento antitrombótico }\end{array}$ \\
\hline Edad & Factores de riesgo & Recomendación \\
Menor de 65 años & Ausentes & Aspirina \\
& Presentes & Warfarina \\
$65-75$ años & Ausentes & Aspirina o warfarina \\
& Presentes & Warfarina \\
Mayor de 75 años & Ausentes o presentes & Warfarina \\
\hline
\end{tabular}


La dosis usual de inicio de la terapia anticoagulante con warfarina es de $5 \mathrm{mg}$, sin embargo, dosis menores podrían ser apropiadas para pacientes mayores de 75 años, desnutridos, hepatópatas y otros con alto riesgo de sangrado ${ }^{45}$. Esta dosis produce efecto anticoagulante observable usualmente entre 2 y 7 días después de iniciada la terapia y se recomienda monitorear el INR diariamente, hasta que alcance un nivel de entre 2 y 3 y luego de 2 a 3 veces por semana, hasta alcanzar estabilidad, momento a partir del cual se podrá controlar el INR cada mes. En el caso de la aspirina, la dosis recomendada varía entre $125 \mathrm{mg}$ y $325 \mathrm{mg}$ por día ${ }^{5}$.

Los efectos adversos más comunes relacionados con el uso de la warfarina son: sangrado, diarrea, alopecia, necrosis intestinal, necrosis cutánea y urticaria ${ }^{44}$, los cuales deben tenerse presentes siempre que se usen como terapia, al igual que sus contraindicaciones.

\section{MANEJO DE LA FA CON CADIOVERSIÓN ELÉCTRICA (ver Figura I)}

Debe realizarse de manera urgente en todo paciente hemodinámicamente inestable y considerarse de forma electiva en todo paciente hemodinámicamente estable. Debe obtenerse antes el consentimiento informado y sedar al paciente; además, debe haber disponibilidad de un equipo completo de reanimación. El procedimiento está indicado en FA no autolimitada, cuando hay necesidad de tratamiento urgente por inestabilidad hemodinámica, fallo de la cardioversión farmacológica, o como primera opción terapéutica.

La mayoría de los autores favorece el uso de la cardioversión eléctrica, debido a que es más rápida y efectiva que la farmacológica. Todo paciente debe ser anticoagulado 3-4 semanas antes del procedimiento, y al menos por 4 semanas después de este, excepto en los pacientes con FA de menos de 48 horas de evolución ${ }^{1,3,13,41,43}$, o en aquellos en que exista disponibilidad de un ecocardiograma transesofágico y se descarte la presencia de trombos en el atrio izquierdo, los cuales se pueden cardiovertir iniciando la warfarina de inmediato en conjunto con heparina, y continuandola por cuatro semanas luego de la cardioversión exitosa.

Igualmente, como se describió para la cardioversión farmacológica, el factor más importante para el éxito, es el tiempo de evolución de la arritmia y se requieren hasta 300 joules para lograr la cardioversión ${ }^{13}$. Esta demostrado que las descargas bifásicas rectilíneas son más eficaces que las monofásicas utilizadas antes ${ }^{8,10}$. Dentro de los efectos adversos de este procedimiento están: las extrasístoles ventriculares, la taquicardia ventricular sostenida y no sostenida, la hipotensión, el edema pulmonar agudo, y el supradesnivel transitorio del segmento S-T.

\section{Otros procedimientos:}

\section{a- Ablación focal con catéter de radiofrecuencia}

La localización de focos de ectopia, principalmente en las venas pulmonares, por medio de estudios electrofisiológicos, permite realizar ablación con catéter de radiofrecuencia. Se considera que los focos en las venas pulmonares podrían ser la causa del $80 \%$ al $95 \%$ de los sitios de ectopia, seguida de focos en las venas cavas, los atrios y el seno coronario ${ }^{46-48}$. Estos focos tienen su origen en extensiones de tejido muscular atrial dentro de las estructuras mencionadas, las cuales tienen un origen embrionario semejante al tejido de conducción del corazón, con automatismo y actividad eléctrica inducida, que les permite funcionar como focos arritmogénicos.

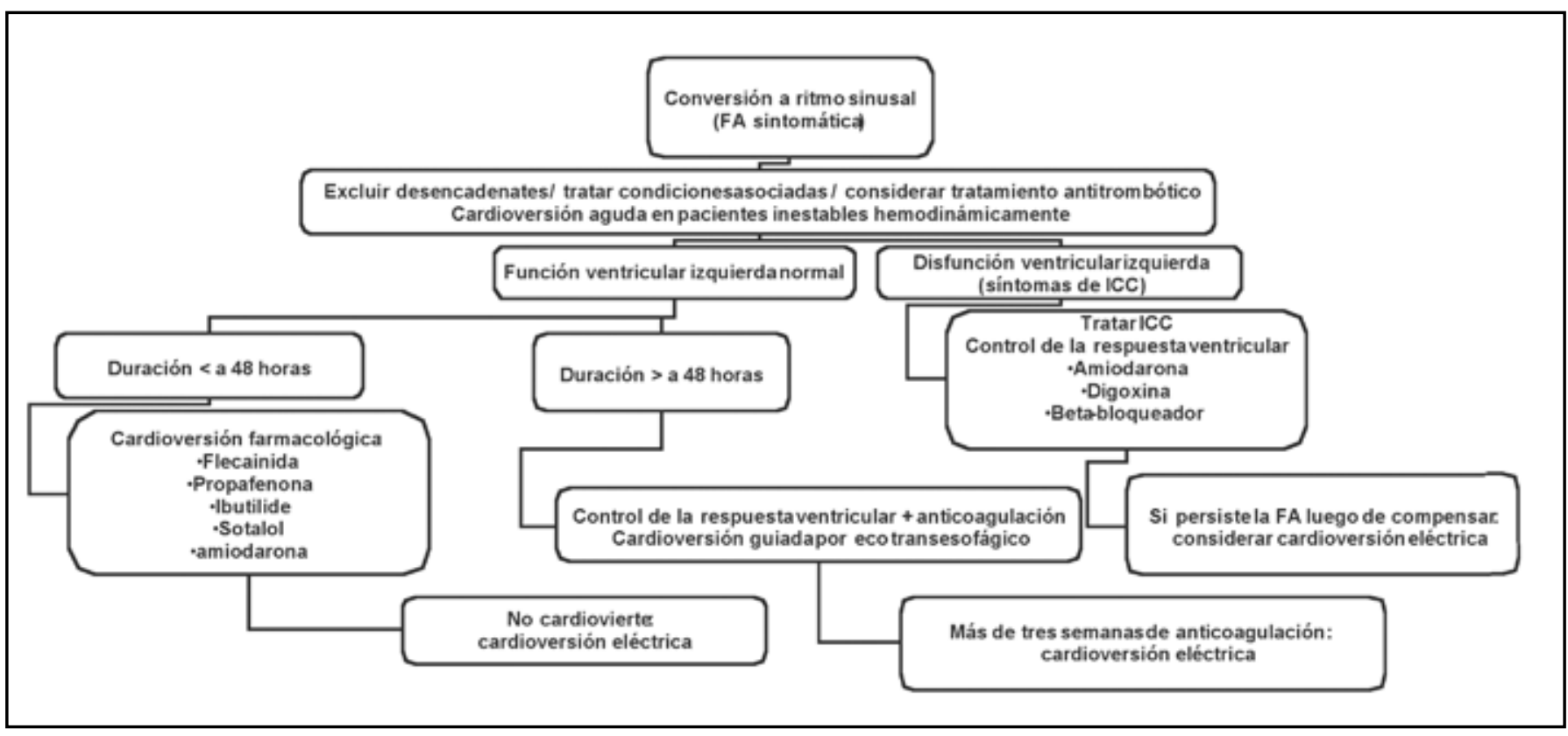

Figura 1. Estrategia de tratamiento para la cardioversión de la fibrilación atrial ${ }^{8}$ 
La ablación se puede realizar de varias maneras:

a. Focal: consiste en localizar el sitio de origen de la arritmia directamente dentro de las venas pulmonares y eliminarlo; este método tiene altos índices de recurrencia y modestos índices de éxito.

b. Aislamiento de las venas pulmonares: se refiere a la ablación circunferencial del ostium de estas. Tiene la ventaja teórica de que aísla focos múltiples y evita la necesidad de mapeo electrofisiológico para la localización del foco de ectopia.

c. Aislamiento segmentario de las venas pulmonares, se basa en que anatómicamente los haces musculares no se extienden desde el atrio a través de toda la circunferencia de las venas pulmonares, sino que están presentes solo en algunos segmentos. Esto permite realizar la ablación únicamente en los segmentos con actividad eléctrica producto de la presencia de fibras musculares, evitando la ablación de toda la circunferencia de las venas pulmonares y reduciendo el riesgo de complicaciones, principalmente la estenosis de las venas pulmonares.

Dentro de las complicaciones más comunes de los procedimientos de ablación se encuentran: derrame pericárdico, episodios de isquemia transitoria, estenosis de las venas pulmonares en el $40 \%$ de los casos, efectos vagales transitorios y embolia sistémica ${ }^{12,47,48}$.

\section{b- Marcapaso atrial para la prevención o terminación de la fibrilación atrial}

El uso de marcapaso atrio-atrio-inhibido (AAI) ha demostrado ser superior que el ventrículo-ventrículo-inhibido (VVI), en pacientes con síndrome del seno enfermo para reducir la incidencia de fibrilación atrial. A su vez, algunos marcapasos con sistema para detección y defibrilación podrían tener un rol importante en la detección y terminación de la arritmia ${ }^{8}$. En pacientes con FA vagotónica, el uso de marcapaso es eficaz si falla el tratamiento antiarrítmico con disopiramida ${ }^{47}$.

En los pacientes postoperados de cirugía de corazón, el uso de marcapaso simultáneo en los dos atrios reduce la incidencia de la FA durante el postoperatorio ${ }^{8,9}$.

\section{c- Desfibrilador atrial implantable}

Este tipo de dispositivos permiten eliminar de forma segura y rápida la $\mathrm{FA}$ en el $80 \%$ de los casos. Los efectos adversos más frecuentes son: recurrencia de la arritmia, dolor por las descargas y problemas técnicos. Los candidatos ideales son pacientes con episodios sintomáticos infrecuentes de la arritmia, que son refractarios al tratamiento antiarrítmico ${ }^{8,13,18,47}$.

\section{d- Ablación del nodo atrioventricular asociado a la coloca- ción de marcapaso}

Este procedimiento consiste en la ablación transvenosa con catéter de radiofrecuencia del nodo atrioventricular y colocación de marcapaso, ya sea atrial o bicameral. Los marcapasos bicamerales han demostrado reducir en mayor grado la incidencia de la FA en comparación con los unicamerales ${ }^{17}$. Aunque la ablación del nodo atrioventricular y colocación de marcapaso no elimina la FA, mejora la calidad de vida, disminuye la sintomatología, aumenta la tolerancia al ejercicio y mejora la función ventricular 13,22,24,47.

Parece ser apropiado en pacientes con FA sintomática y respuesta ventricular no controlada, en quienes la terapia con múltiples drogas ha fallado, particularmente si hay evidencia de miocardiopatía inducida por taquicardia ${ }^{8,47}$

\section{e- Procedimiento de Maze III}

Esta cirugía introducida por Cox en $1987^{8,13}$, consiste en la realización estratégica de incisiones en el atrio, eliminación de los apéndices auriculares y aislamiento de las venas pulmonares, con la finalidad de producir aislamiento de los múltiples circuitos de reentrada, previniendo o terminado la FA ${ }^{47,50}$. Ha demostrado mejorar la calidad de vida de los pacientes a quienes se les realizó ${ }^{11}$.

El procedimiento realizado satisfactoriamente elimina la arritmia, no produce anomalía en la función del nodo sinusal, no posee mortalidad y acarrea una baja morbilidad ${ }^{51}$. Este procedimiento es de gran utilidad en pacientes con FA que son operados para corrección de enfermedad arterial coronaria o cardiopatía valvular ${ }^{8,47}$

\section{f- Cirugía de Batista o reducción del tamaño del atrio izquierdo ${ }^{52}$}

Este procedimiento usualmente se realiza en el mismo tiempo quirúrgico que un reemplazo valvular mitral, en pacientes que han tenido fibrilación atrial por más de un año.

Luego de la división de la vena cava superior, el atrio izquierdo es seccionado circunferencialmente, permitiendo la rotación del corazón dentro del campo operatorio y generando una excelente exposición de la válvula mitral, la cual es reparada o reemplazada. Posteriormente, un anillo de 1 ó 2 centímetros de tejido del atrio izquierdo, incluyendo el apéndice de este son removidos; luego el atrio es reconstruido.

Un estudio preliminar de Winlaw y colaboradores, demostró recuperación del ritmo sinusal en todos los casos, con respuesta sinoatrial normal al ejercicio, sin que se requiriera el uso de marcapaso permanente. 


\section{FIBRILACIÓN ATRIAL POSTERIOR A CIRUGÍA CARDIOTORÁCICA}

La fibrilación atrial es la taquiarritmia más común en el postoperatorio de cirugía a corazón, presentándose en el $10 \%$ al $53 \%$ de los pacientes ${ }^{53-56}$, y usualmente en el segundo día postoperatorio; a su vez representa una causa importante de prolongación de la estancia hospitalaria y costos excesivos postcirugía.

Dentro de los factores de riesgo para desarrollarla, el más importante es la edad ${ }^{54}$, sin embargo, se postulan como probables predisponentes, la suspensión de los bloqueadores beta, el uso de circulación extracorpórea, inadecuada protección atrial y excesiva manipulación del atrio ${ }^{54}$.

Los estudios clínicos sobre tratamiento profiláctico con verapamilo y la digoxina no demostraron beneficio para reducir esta complicación ${ }^{56}$, mientras que un meta-análisis de estudios con bloqueadores de los receptores beta, han demostrado beneficio para la prevención de la FA postoperatoria, y estos deben considerarse como fármacos de primera línea ${ }^{57}$.

Actualmente se postula el uso de amiodarona o sotalol durante los siete días preoperatorios, continuando la terapia en el postoperatorio con la finalidad de reducir la incidencia de la arritmia. Los estudios demuestran que ninguno de los dos regímenes es superior al otro ${ }^{55}$, pero reducen la prevalencia de la FA en un $21.5 \%$ el sotalol y un $14.1 \%$ la amiodarona, ${ }^{55}$ además, otro estudio demostró reducción del $6 \%$ de la FA, en pacientes que recibieron amiodarona a partir del postoperatorio inmediato, evitando el monitoreo de los pacientes externos que inician el tratamiento siete días precirugía ${ }^{56}$. La amiodarona tiene como ventajas su metabolismo hepático, que no reduce la función ventricular izquierda y no causa broncoespamo, lo cual lo convierte en una excelente opción en pacientes con insuficiencia renal, enfermedad pulmonar obstructiva crónica y con disfunción sistólica del ventrículo izquierdo; ambos fármacos deben considerarse como alternativas apropiadas.

Otro estudio demostró que el uso profiláctico de marcapaso simultáneo, biatrial en modo disparado, o provocado luego de cirugía a corazón abierto, reduce en un $60 \%$ la prevalencia de la FA, siendo además bien tolerado y sin elevar las complicaciones postoperatorias ${ }^{49}$.

\section{Conclusión}

La fibrilación atrial es la arritmia más común en la práctica clínica, y se presenta tanto en pacientes con enfermedad cardiaca de fondo, como en ausencia de esta. Su espectro clínico puede variar desde una arritmia de inicio súbito con gran inestabilidad hemodinámica, hasta un hallazgo asintomático clínico o electrocardiográfico.

Los múltiples estudios realizados en la década anterior en el manejo de la anticoagulación, el uso de antiarrítmicos, los procedimientos electrofisiológicos de ablación, el empleo de marcapaso y las diversas técnicas quirúrgicas, han permitido disponer de un mayor conocimiento de las implicaciones en la calidad de vida de los pacientes que la sufren, lo mismo en cuanto a morbimortalidad asociada y los costos en salud que su manejo conlleva.

Este interés en el estudio de la arritmia ha permitido que hoy en día el manejo de los pacientes que la padecen sea estandarizado y fundamentado en la medicina basada en la evidencia, sin embargo, todavía existen muchos aspectos de la fibrilación atrial que no se conocen y que sin duda serán aclarados en los años siguientes.

\section{A bstract}

Atrial fibrillation is the most frequent arrhythmia seen in clinical practice. ${ }^{1-12}$ It occurs most commonly on patients with hypertensive, isquemic and valvular heart disease as well as in patients without cardiac disease principally in elderly patients. Due to the morbidity rate associated with it (atrial fibrillation), early diagnosis and adequate treatment is necessary in order to reduce any associated complication and to improve the health related quality of life of patients who suffer from it.

\section{Referencias}

1. Ukani ZA, Ezekowitz MD. Tratamiento actual de la fibrilación auricular. Clin Med NA 1995;5: 1119-1138.

2. Hohnloser SH, Kuck K, Lilienthal J. Rhythm or rate control in atrial fibrillation-pharmacological intervention in atrial fibrillation (PIAF): a randomised trial. Lancet 2000;356: 1789-94.

3. Klein AL, Grimm RA, Murray D, Apperson-Hansen C, Asinger RW, Black IW, et al. Use of transesophageal ecocardiography to guide cardioversion in patients with atrial fibrillation. N Engl J Med 2001;344: 1411-20.

4. Roy D, Talajic M, Dorian P, Connolly S, Eisenberg MJ, Green M, et al. Amiodarone to prevent recurrence of atrial fibrillation. N Engl J Med 2000;342: 913-20.

5. Albers GW, James DE, Laupacis A, Manning WJ, Palle P, Singer DE. Antithrombotic therapy in atrial fibrillation. Chest 2001;119: 194s206s.

6. Silverman DI, Manning WJ. Role of echocardiography in patients undergoing elective cardioversion of atrial fibrillation. Circulation 1998;98:479-486.

7. Markides V, Schilling RJ. Atrial Fibrillation: classification, pathophysiology, mechanisms and drug treatment. Heart 2003;89: 939-943.

8. Blaauw Y, Van Gelder IC, Crijns HJGM. Treatment of atrial fibrillation. Heart 2002;88:432-437.

9. Madrid AH, Bueno MG, Rebollo JMG, Marín I, Peña G, Bernal E, et al. Use of irbersartan to maintain sinus rhythm in patients with long-lasting persistent atrial fibrillation: a prospective and randomized study. Circulation 2002;106:331-336.

10. Mittal S, Ayati S, Stein KM, Schawartzman D, Cavlovich D, Tchou PJ, et al. Transthoracic cardioversion of atrial fibrillation: comparison of rectilinear biphasic versus damped sine wave monophasic shocks. Circulation 2000;101:1282-1287. 
11. Lönnerholm S, Blomström P, Nilsson L, Oxelbark S, Jideus L, Blomström-Lundqvist C. Effects of the maze operation on healthrelated quality of life in patients with atrial fibrillation. Circulation 2000;101:2607-2611.

12. Wyndham CRC. Atrial fibrillation: the most common arrhytmia. Tex Heart Inst J 2000;27:257-267.

13. Falk RH, Atrial fibrillation. N Engl J Med 2001;344:1067-78.

14. Arango JJ. Manual de electrocardiografía. Cap.19. Taquiarritmias. Corporación para investigaciones biológicas. Colombia. 1996, p:205-208.

15. Josephson ME, Zimetbaum P, Buxton AE, Marchlinsk FE. Harrison: principios de Medicina Interna. Cap. 231. Las taquiarritmias. Mc Graw Hill. México. 1999, p:1446-1447.

16. Robles EO, Algra A. Digoxin in the treatment of paroxysmal atrial fibrillation. Lancet 1999;354:882-83.

17. Cooper JM, Katcher MS, Orlov MV. Implantable devices for the treatment of atrial fibrillation. N Engl J Med 2002;346:2062-2068.

18. Haissaguerre M, Jais P, Shah DC, Takahashi A, Hocini M, Quiniou G, et al. Spontaneous initiation of atrial fibrillation by ectopic beats origunating in the pulmonary veins. N Engl J Med 1998;339:659-66.

19. Almendral J, Marín E, Medina O, Peinado R, Pérez L, Ruiz R, et al Guía de práctica clínica de la sociedad española de cardiología de arritmias cardiacas. Rev Esp Cardiol 2002;54:307-67.

20. The atrial fribrillation follow-up investigation of rhythm manegement (AFFIRM) investigators. A comparision of rate control and rhythm control in patientes with atrial fribrillation. N Engl J Med 2002;347:183533.

21. Berge E, Abdelnoor M, Nakstad PH, Sandset PM. Low molecularweight heparin versus aspirin in patients with acute ischaemic stroke and atrial fribrillation: a double-blind randomised study. Lancet 2000;355:1205-10.

22. Ozcan C, Jahangir A, Friedman PA, Patel PJ, Munger TM, Rea RF, et al. Long-term survival after ablation of the atrioventricular node and implantation of a permanent pacemaker in patients with atrial fibrillation. N Engl J Med 2001;344:1043-51.

24. Klein I, Ojamaa K. Thyroid hormone and the cardiovascular system. N Engl J Med 2001;344:501-9.

25. Allessie MA, Boyden PA, Camm AJ, Kléber AG, Lab MJ, Legato MJ, et al. Pathophysiology and prevention of atrial fibrillation. Circulation 2001;103:769-777.

26. Morady F. Radio-frequency ablation as treatment for cardiac arrhythmias. N Engl J Med 1999;340:534-44.

27. Feng TC, Tai CT, Hsieh MH, Lin WS, Yu WC, Ueng KC, et al. Initiation of atrial fibrillation by ectopic beats originating from superior vena cava: electrophysiological characteristics and results of radiofrequency ablation. Circulation 2001;102:67-74.

28. Huikuri HV, Castellanos A, Myerburg RJ. Sudden death due to cardiac arrhythmias. N Engl J Med 2001;345:1473-82.

29. Zipes DP. Braunwald: Tratado de Cardiología. Cap. 22. Arritmias específicas: diagnóstico y tratamiento. Mc Graw Hill. México, p:708-711.

30. Andreoli TE, Bennett JC, Carpenter CCJ, Plum F. Cecil: Compendio de Medicina Interna. Cap. 8. Arritmias. Mc Graw Hill. México. 1999, p:68-92.

31. Bigger JT. Cecil: Tratado de medicina interna. Cap. 35. Arritmias cardiacas. Mc Graw Hill. México. 1999, p:266-277.

32. Iturralde P. Arritmias cardiacas. Cap. 7. Fibrilación y Flúter auricular. Mc Graw Hill. México. 1997, p:119-144.

33. Alpert MA. Medical cardioversion of atrial fribrillation. Chest 2000;117:1530-31.

34. Sánchez K, Padilla J. Manual de enfoque práctico en cuidados intensivos. En diagnóstico y tratamiento de las taquiarritmias. Editorial de la Universidad de Costa Rica. Costa Rica 2000, p.86-92.
35. Giri S, White CM, Dunn AB, Felton K, Freeman-Bosco L, Reddy P, et al. Oral amiodarone for prevention of atrial fibrillation after open heart surgery, the atrial fibrillation suppression trial (AFIST): a randomised placebo-controlled trial. Lancet 2001;357:830-836.

36. Curtis AB. New insights into the pharmacological management of atrial fibrillation. Medscape 2003.

37. Stambler BS, Wood MA, Ellebogen KA, et al. Efficacy and safety of repeated intravenous doses of ibutilide for rapid conversion of atrial flutter or fibrillation. Ibutilide repeat dose study investigators. Circulation 1996;94:1613-21.

38. Go AS, Hylek EM, Phillips KA, Borowsky LH, Henault LE, Chang Y, et al. Implications of stroke risk criteria on the anticoagulation decision in nonvalvular atrial fibrillation: the anticoagulation and risk factors in atrial fibrillation (ATRIA) study. Circulation 2000;102:11-13.

39. Blackshear JL, Baker VS, Rubino F, Safford R, Lane C, Flipse T, et al. Adjusted-dose warfarin versus low-intensity, fixed-dose warfarin plus aspirin for high-risk patients with atrial fibrillation: stroke prevention in atrial fibrillation III randomised clinical trial (SPAF III). Lancet 1996;348:633-38.

40. Lip GYH. Thromboprophylaxis for atrial fibrillation. Lancet 1999;353:4-5.

41. Hammill SC, Hubmayr RD. The rapidly changing management of cardiac arrhythmias. Am J Respir Crit Care Med 2000;161:1070-73.

42. Sudlow M, Thomson R, Thwaites B, Rodgers H, Kenny RA. Prevalence of atrial fibrillation and eligibility for anticoagulants in the community. Lancet 1998;352:1167-71.

43. Gornick Ch. Anticoagulant use in nonvalvular atrial fibrillation. Postgrad Med 2000;108:113-26.

44. O’Reilly RA. Katzung: Farmacología básica y clínica. Cap. 34. Fármacos en los trastornos de la coagulación. Manual Moderno. México. 1999,p:640-643.

45. Ansell J, Hirsh J, Dalen J, Bussey H, Anderson D, Poller L, et al. Managing oral anticoagulant therapy. Chest 2001;119:22s-38s.

46. Peters NS, Schilling RJ, Kanagaratnam PV, Markides V. Atrial fibrillation: strategies to control, combat, and cure. Lancet 2002;359:593603.

47. Scheinman MM, Morady F. Nonpharmacological approaches to atrial fibrillation. Circulation 2001;103:2120-2125.

48. Grant AO. Recent advances in the treatment of arrhythmias. Circ $\mathrm{J} ; 67: 651-655$.

49. Daoud EG, Dabir R, Archambeau M, Morady F, Strickberger A. Randomized, double-blind trial of simultaneous right and left atrial epicardial pacing for prevention of post-Open heart surgery atrial Fibrillation. Ciculation 2000;102:761-765.

50. Galloway AC, Anderson RU, Brossi EA, ESsencer FC, Colvin SB Schwartz: principios de cirugía. Cap. 18. Cardiopatías adquiridas. Mc Graw Hill. México, p:964-965.

51. Jessurun ER, van Hemel NM, Defauw J, Stofmeel M, Kelder JC, de la Rivière $A B$, et al. Results of maze surgery for lone paroxysmal atrial fibrillation. Circulation;101:1559-1567.

52. Winlaw DS, Farnsworth AE, Macdonald PS, Mundy JA, Spratt PM. Left atrial reduction: the forgotten Batista. Lancet 1998;351:879-80.

53. Johnson F. Atrial fibrillation after coronary artery bypass grafting. The Internet Journal of Cardiology 2002;1:1-3.

54. Wurderman RL, Mooss AN, Mohiuddin SM, Lenz TL. Amiodarone vs sotalol as prophylaxis against atrial fibrillation/flutter after heart surgery: a meta-analysis. Chest 2002;121:1203-1209.

55. Stamou SC, Hill PC, Sample GA, Snider E, Pfister AJ, Lowery RC, et al. Prevention of atrial fibrillation after cardiac surgery: the significance of postoperative oral amiodarone. Chest 2001;120:1936-1941.

56. Crystal E, Connolly SJ, Sleik K, Ginger TJ, Yusuf S. Interventions on prevention of postoperative atrial fibrillation in patients undergoing heart surgery: a meta-analysis. Circulation 2002;106:75-80. 